\title{
The Role of Carbon Dioxide Laser Therapy in Penile Kaposi's Sarcoma: A Case Series of Three HIV-Negative HHV-8-Positive Patients
}

Tullio Torelli ${ }^{1}$, Biagio Paolini ${ }^{2}$, Mario Achille Catanzaro ${ }^{1}$, Nicola Nicolai ${ }^{1}$, Maurizio Coleccloia $^{2}$, Davide Biasoni ${ }^{1}$, Alberto Macchi ${ }^{1}$, Silvia Stagni ${ }^{1}$, Antonio Tesone ${ }^{1}$, Roberto Salvioni ${ }^{1}$

\section{Abstract}

Kaposi's sarcoma $(K S)$ is a rare tumor of vascular origin. It is quite common in HIV patients. It is rarely located on the glans penis, especially in HIV-negative patients (just some tens in the literature). Owing to the rarity of the disease, both the real impact on survival and the most suitable therapy are not known.

However, in this 3-patient case series, carbon dioxide laser therapy was effective both for local control and survival. In fact, two late relapses but no disease-related death were recorded. Probably, KS in HIV-negative patients is a slowly progressive disease, not so aggressive as in $H I V$-positive patients. Laser therapy is easy, fast, and cheap, and may treat the disease radically. If these data are confirmed by further studies, in the foreseeable future, laser therapy may become the gold standard for treating $H I V$-negative patients affected by penile $K S$.

Keywords: Penile Neoplasms; Laser Therapy; Sarcoma, Kaposi; Herpesvirus 8, Human; Infection

CMI 2019; 13(1): 11-17

https://doi.org/10.7175/cmi.v13i1.1403

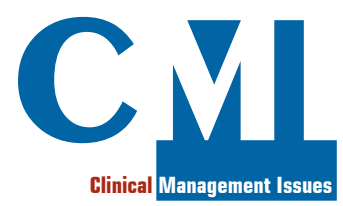

Case Series
${ }^{1}$ Department of Urology,

Fondazione IRCCS

Istituto Nazionale

Tumori, Milan, Italy

${ }^{2}$ Department of Pathology,

Fondazione IRCCS

Istituto Nazionale

Tumori, Milan, Italy

\section{INTRODUCTION}

Kaposi's sarcoma (KS) is a lesion of vascular origin, known since the $19^{\text {th }}$ century. To date, four different clinical forms have been identified [1]:

- the classic, which is typical in Mediterranean countries and the central regions of Africa;

- the lymphoadenopathic, a form that occurs in young African children;

- the transplantation-associated, particularly affecting patients who underwent kidney transplantation;

- the AIDS-related.

Kaposi's sarcoma affects primarily the skin, but it may also be detected in the visceral mucosa, even if it is rarely located on the glans penis ( 37 cases found in the literature, according to Rescigno and colleagues [2]), sometimes involving HIV-negative patients (in 2003, Micali et al. [3] calculated 19 patients in the literature; afterwards, just a few isolated case reports were published).

In 1994, Chang and colleagues discovered the association between $\mathrm{KS}$ and infection of neoplastic cells by human herpes virus type 8 (HHV-8) [4]. This virus belongs to the family of gamma-herpesviruses and its role as a potential oncogenic virus is supported by its tight relationship with primary effusion lymphoma (PEL). The genome of this virus was shown to be present in about $90 \%$ of $\mathrm{HIV}$-positive patients with KS. This implies a role in the etiology of Kaposi's sarcoma.

\section{Why Do we Describe These Cases}

Penile Kaposi's sarcoma is an uncommon disease with a very difficult histopathological diagnosis. To date, the most effective therapy is not known
Corresponding author Tullio Torelli

Department of Urology Fondazione IRCCS Istituto Nazionale Tumori Milan, Italy tullio.torelli@istitutotumori.mi.it

Received: 22 January 2019 Accepted: 21 February 2019 Published: 1 March 2019 


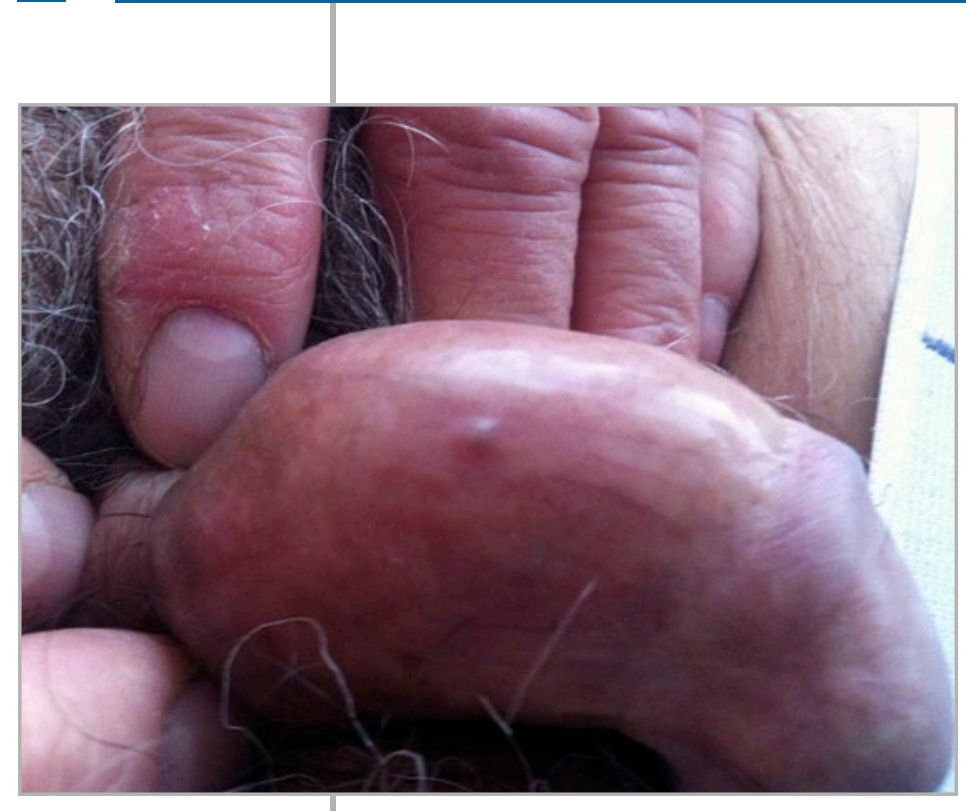

Figure 1. Small and bluish lesion of the dorsal penile cutis before carbon dioxide laser excision (case 2)

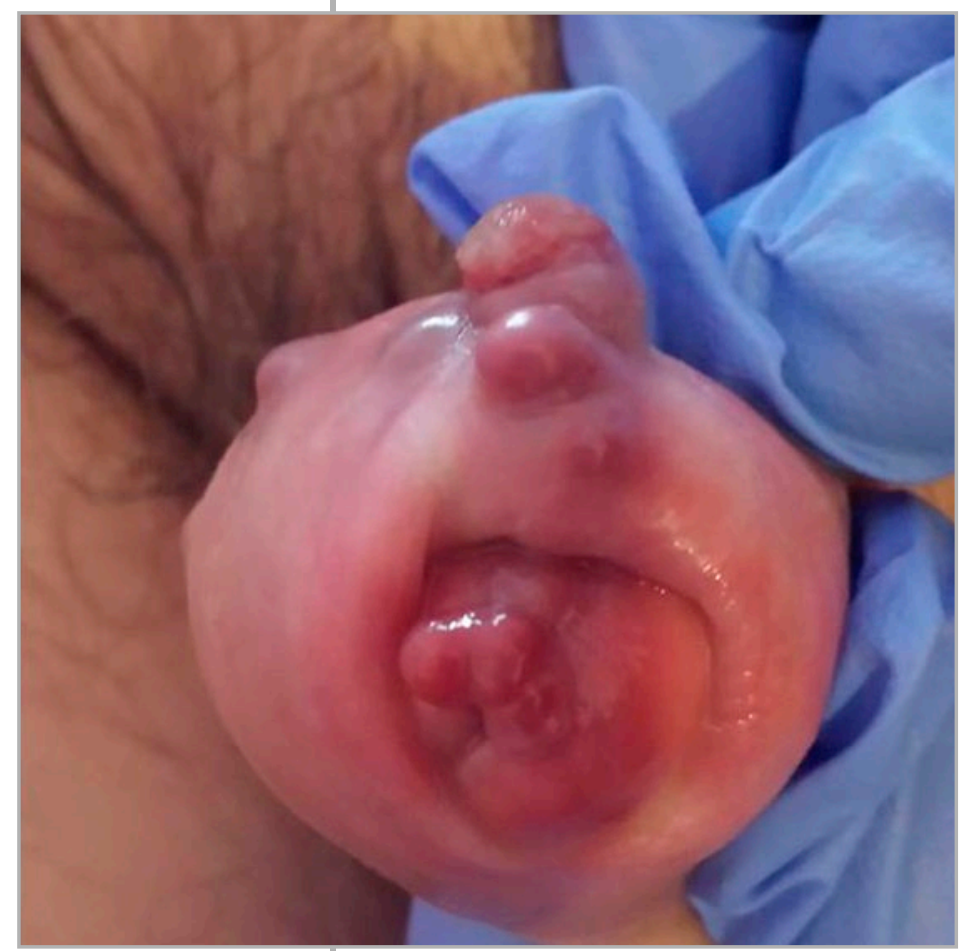

Figure 2. Plurifocal nodular and reddish lesions in the glans and the prepuce before carbon dioxide laser excision and surgical circumcision (case 3)
We report three cases involving HIVnegative patients with lesions on the mucosa of glans penis and penile shaft. Tumor cells were infected by HHV-8.

men CT scans were performed before surgery and after 12 and 24 months. Moreover, outpatient examinations were performed every six months for at least five years. An inguinal ultrasound was performed during examinations.

\section{Case 1}

S.G. was 60 years old, came from Sicily, and underwent a surgical excision of a small lesion of the glans in September 2013 in another hospital. He was diagnosed with KS, but no test for HHV-8 was performed. No major disease was recorded.

In November 2016, he came to the Urologic Unit of our Institute for a mucosal lesion of the dorsal glans. The lesion was radically excised by carbon dioxide laser and he was diagnosed with Kaposi's sarcoma associated HHV-8. HIV tests were negative.

In May 2017, another lesion of the dorsal glans was excised and the diagnosis was the same. Before and after surgery, the disease staging by CT scan was negative and at present the patient is disease-free.

\section{Case 2}

Z.O. was 71 years old, came from Sicily, and underwent a surgical excision of a perimeatal lesion of the glans in July 2013 in another hospital. The surgical excision was performed during a transurethral resection of prostate (TURP) for benign prostatic hyperplasia. No other major disease was recorded. Also in this patient the diagnosis was KS but no test for HHV-8 was performed. $\mathrm{HIV}$ test was negative.

In May 2014, he was examined in the Urologic Unit of our Institute for a small and bluish lesion of the dorsal penile cutis (Figure 1), that was radically excised by carbon dioxide laser. The diagnosis was Kaposi's sarcoma associated HHV-8.

In July 2015, a small preputial lesion (histological relapse of $\mathrm{KS}$ ) was laser excised. Before and after surgery, no systemic disease was found at CT scan and at present the patient is disease-free.

\section{CASES DESCRIPTION}

In our patients, we recorded main diseases and the medical history linked to the penile lesions. Moreover, we analyzed their immune status (particularly, testing for HIV virus) and photos of the lesions were taken before and after surgery. Chest and abdo-

\section{Case 3}

N.F. was 77 years old, came from Apulia, and was examined in our Urologic Unit for plurifocal nodular and reddish lesions of the glans and the prepuce (Figure 2) in July 2014.

He was suffering from diabetes type 2, mild hypertension, and obesity. The lesions 


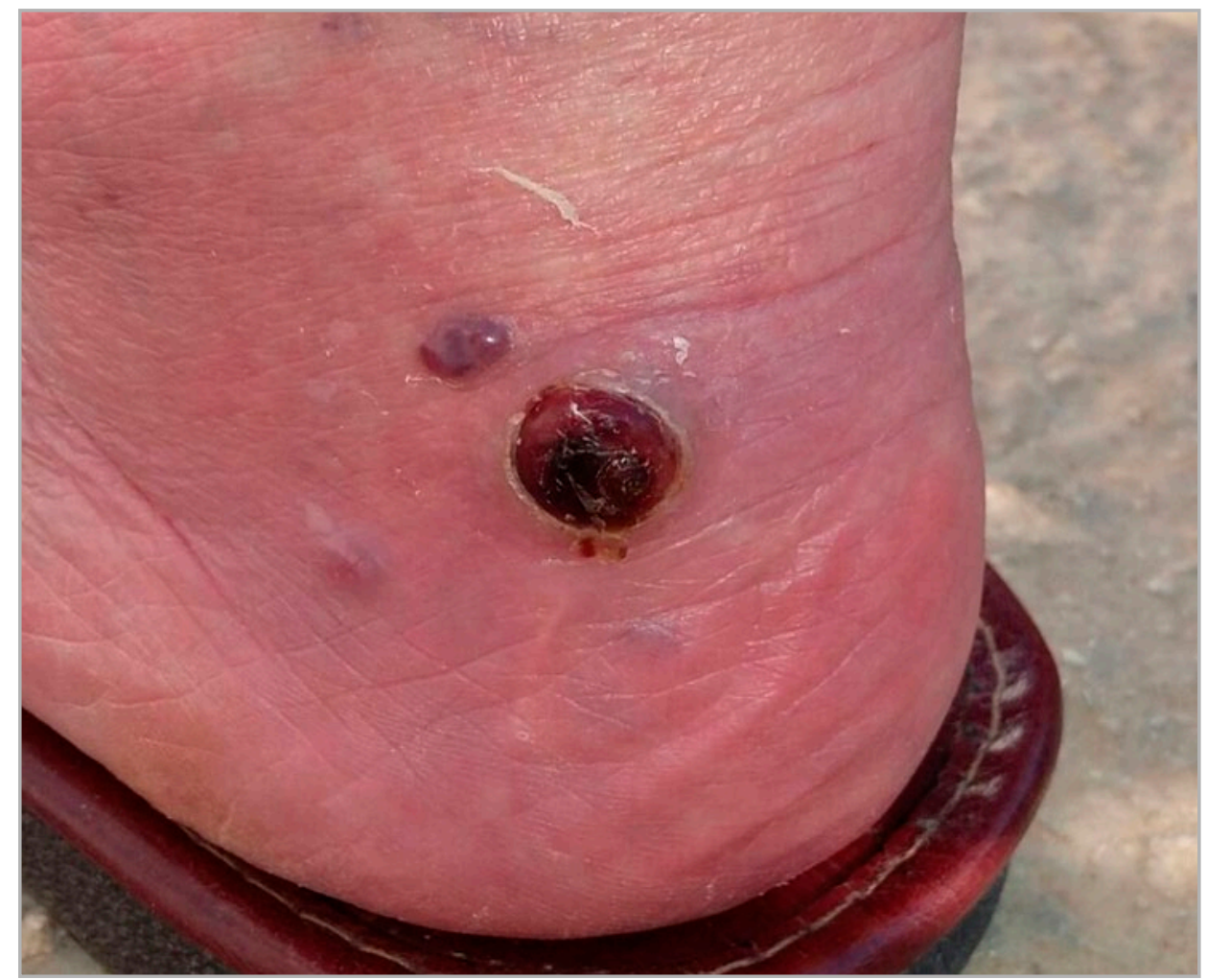

Figure 3. Reddish

papules of the perimalleolar skin found two years after carbon dioxide laser excision of the lesions in the glans and surgical circumcision (case 3)

were radically excised by carbon dioxide laser and surgical circumcision. In every specimen, KS-associated HHV-8 was found. Before surgery, HIV test and CT scan were negative. After surgery, CT scans did not show any systemic disease.

After two years, reddish papules of the perimalleolar skin were found (Figure 3), but they were not removed because the patient suddenly died from stroke. No local relapse was found.

\section{Main surgical aspects}

All the lesions on the glans penis and penile shaft were locally anesthetized with $2 \%$ lidocaine after the local application of anesthetizing gel (EMLA). Ablation was done with a high-energy pulsed carbon dioxide laser at a power of 10 watts with a 4-mm spot size (SHARPLAN $40 \mathrm{C} \mathrm{CO}_{2}$ 40 Watt, $10600 \mathrm{~nm}$, KOSMO). There was very little bleeding during the treatment and the lesions healed rapidly in a few days with topical antibacterial (gentamicin) ointment application, leaving good cosmetic results (Figure 4).

\section{Immunohistochemical analysis}

The biopsy specimen was routinely fixed in formalin (10\%), embedded in paraffin and then cut at $4 \mu \mathrm{m}$ and stained with hematox-

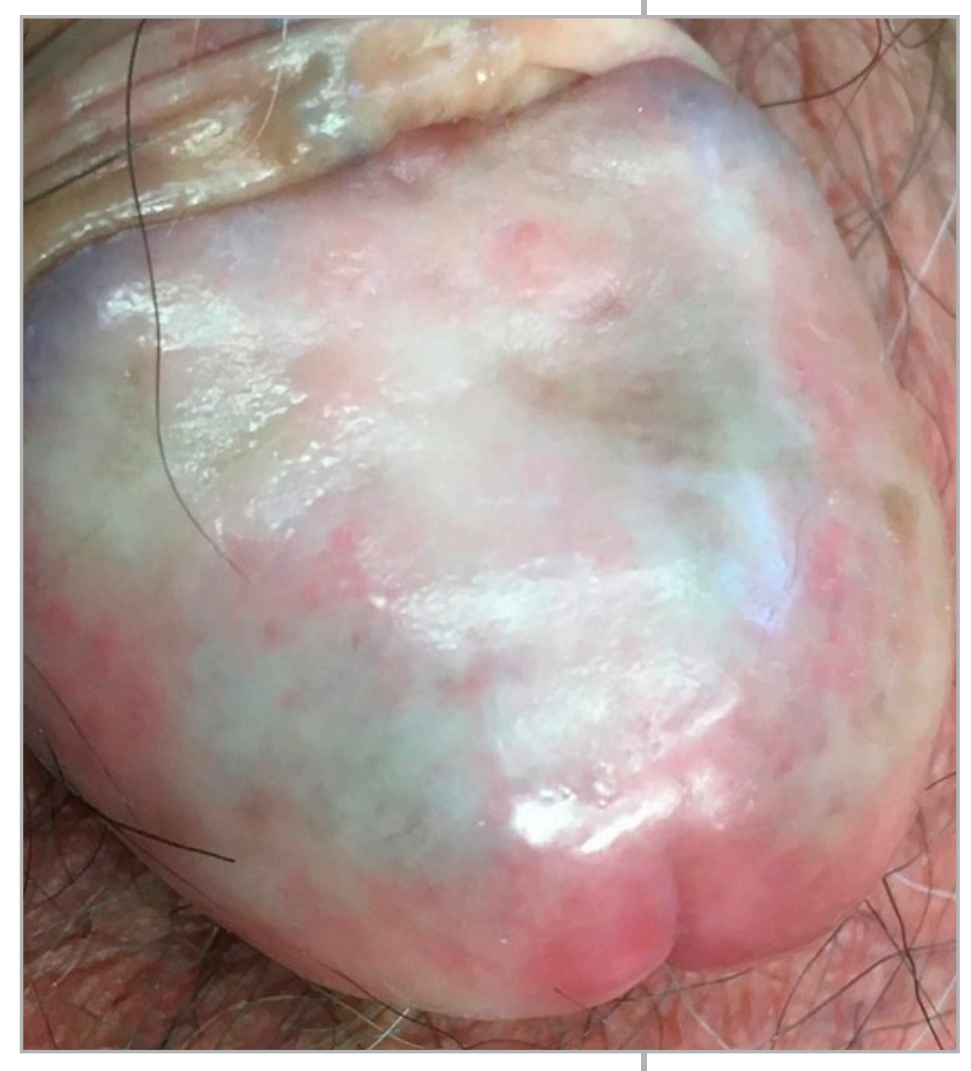

ylin-eosin. The immunohistochemical studies were performed on paraffin tissue sections of the lesion using antibodies against CD34 and factor VIII-related antigen (HHV-8, clone LN53, Novus; DAKO au-
Figure 4. Cosmetic results two years after treatment (case 1) 

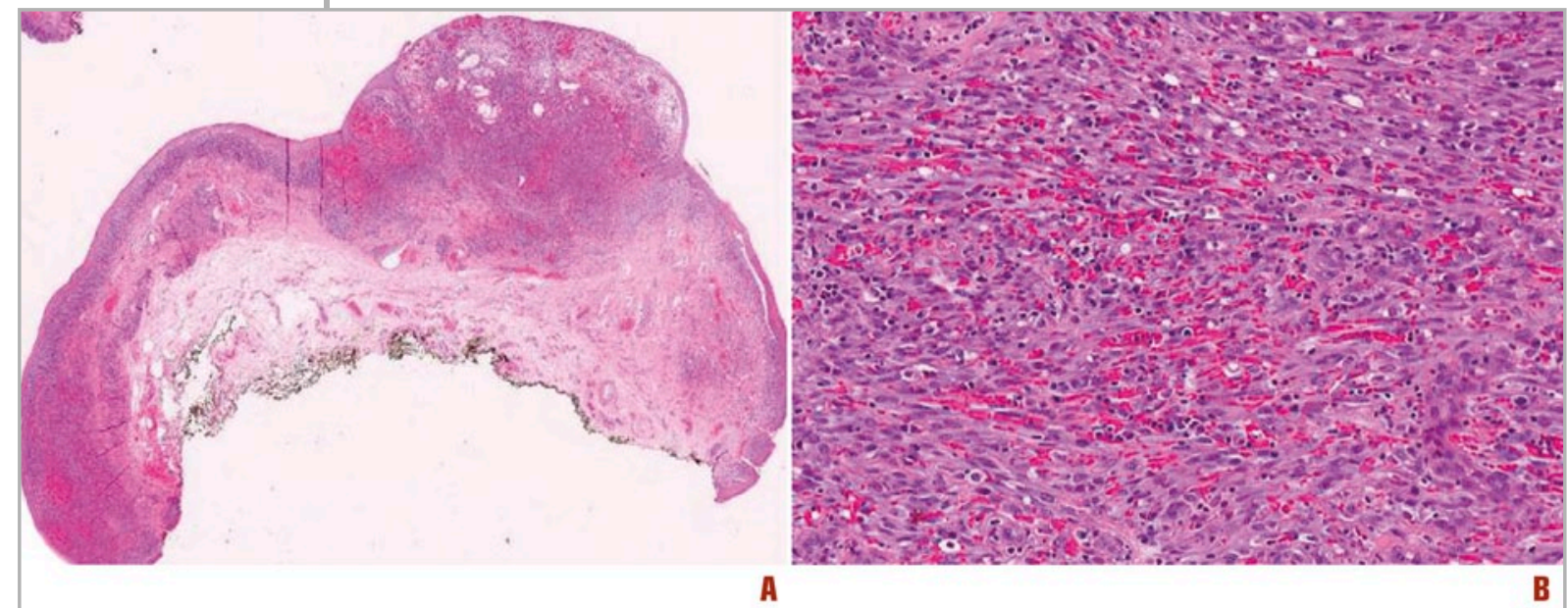

A

B

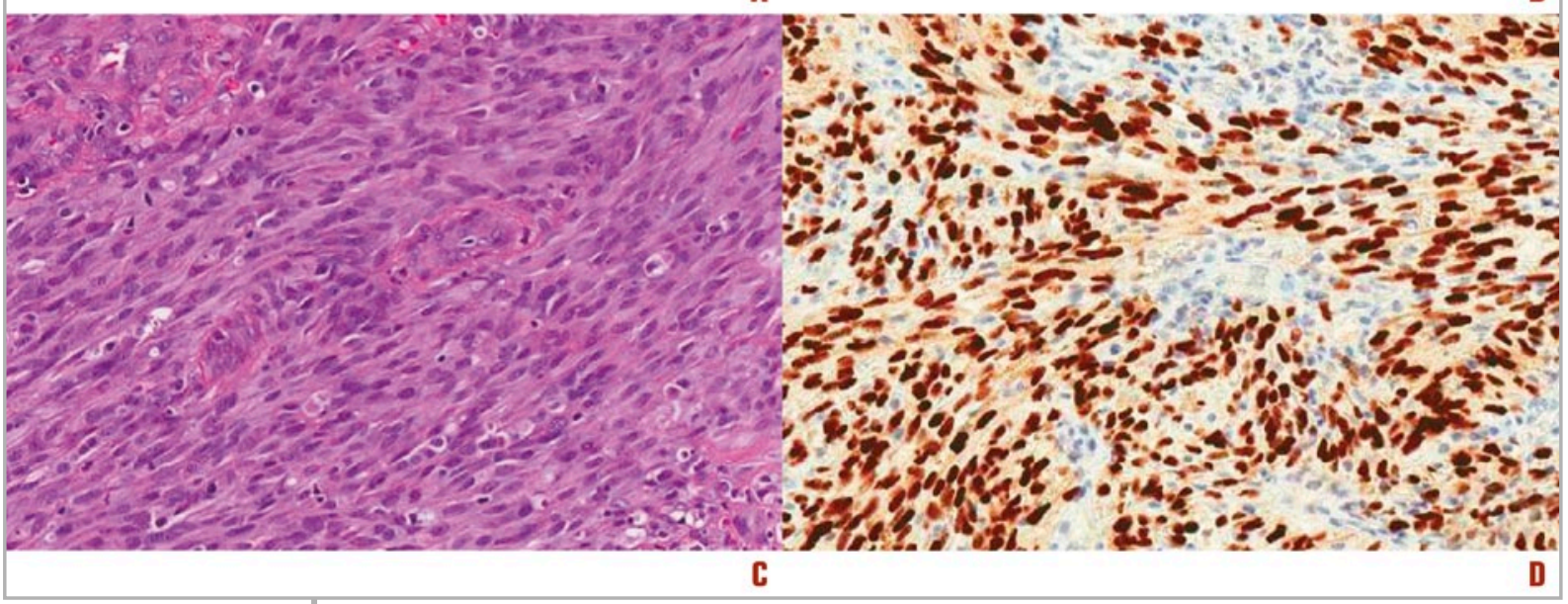

Figure 5.

Immunobistochemical analysis (case 3)

A. Hematoxylin-eosin stain, $0.3 \times$ magnification

B. Hematoxylin-eosin stain, $10 \times$ magnification

C. Hematoxylin-eosin stain, $15 \times$ magnification

D. DAKO autostainer, $15 \times$ magnification tostainer). Histologically, lesions were nodules in the lamina propria, with thinning of the overlying squamous epithelium. Some dilated and irregular vascular channels could be seen in the top of the lesions, while the other parts were formed by more compact cellular proliferation with ill-defined borders (Figure 5A). There were typical spindle cells of the nodular KS with vascular channels formation filled with red blood cells and sprinkling chronic inflammation (Figure 5B). Normally, Kaposi's cells show slight nuclear atypia and very low mitotic count (Figure 5C). Immunohistochemical analysis showed nuclear positivity for $\mathrm{HHV}-8$ only in the neoplastic cells (HHV-8, clone LN53, Novus) but not in the surrounding ones (Figure 5D).

\section{Results}

After surgical excision, the relapse occurred on average after 18 months while, after laser ablation, it occurred after 14 months. However, it should be noted that, while S.G. (case 1) had relapses close to the first localization (glans), the other two pa- tients had new localizations that were far from the first ones: penile skin (case 2) and perimalleolar area (case 3). Two patients were disease-free after 19 and 41 months (mean disease free time: 30 months), while the last one died from stroke after two years from laser ablation, with perimalleolar disease but no local relapses.

\section{DISCUSSION}

Primary penile Kaposi's sarcoma is observed in young patients and it is strongly associated with HIV infection. In immunocompetent patients it is very rare and, in Mediterranean area, it is often present in patients in the sixth and seventh decade, like our aged patients (mean age $=69$ years), who were from Southern Italy, and usually involves the lower extremities, as patient N.F. (case 3) in Figure 3. Penile primary $\mathrm{KS}$ is very rare in healthy males: in English literature, just a few tens of immunocompetent patients are described (even if HIV or HHV-8 tests are not performed in every patient) [3]. 
What Should the Clinician Ask Him/Herself About the Patient

- How long has the penile lesion been going on?

- Is there a history of high-risk sexual intercourses?

- Are there general symptoms as asthenia, malaise, and mild fever as in HIV patients?

The pathogenesis of KS is still uncertain, but recent data showed a strong correlation between all forms of $\mathrm{KS}$ and $\mathrm{HHV}-8$ infections. This virus, discovered in 1994, plays a role as a potential oncogenic virus and is similar to two other oncogenic herpes viruses (Saimiri and Epstein-Barr virus) in terms of ability to alter the growth of human endothelial cells in vitro. High HHV-8 seroprevalence in individuals with high-risk sexual activity or HIV-seropositive patients denotes the route of sexual transmission, while the detection of $\mathrm{HHV}-8$ antibodies in children also suggests a non-sexual route: saliva could be a potential source of spread in general population. Notably, HHV-8 is endemic in Sicily [5] and all our patients were from Southern Italy (two from Sicily and one from Apulia). Moreover, they did not claim high-risk sexual activity and had few partners before marriage.

Among patients with classic KS, primary penile lesions are usually single reddishpurple to bluish nodules (case 2, Figure 1). Multiple papules, nodules (case 3, Figure 2), plaques, and wart-like or pedunculated lesions are less common presentations. The most frequently involved site is the glans, sometimes in association with swelling and lymphatic edema due to massive involvement. Lesions may also involve the foreskin, the coronal sulcus, the meatus and, rarely, the shaft [6].

Local therapies for KS include simple excision, cryotherapy, radiation [7], and intralesional therapy. Simple excision is seldom used because it is frequently unsatisfactory [8]. Cryotherapy is mostly effective for small lesions, with $85 \%$ of clinical response. On the other hand, radiotherapy may be useful in large scrotal and penile lesions, but it is a time-consuming process [9]. A 50\% decrease or greater was achieved in $62 \%$ of lesions for intralesional vincristine [10], 50 to $90 \%$ for intralesional interferon alpha-2 [11], 56\% for imiquimod [12]. However, the evidence of efficacy of any particular therapy is of low quality and does not support recommending any particular choice. Ultimately, treatment guidelines are lacking for classic Kaposi sarcoma in English literature [13].
Laser therapy is seldom used: in English literature only one case was found, dating back to 1999 [14]. Carbon dioxide laser has not been popular because of the possible risk of infectious viral particles in the vapor plume. However, our patients have no major diseases, particularly they have no evidence of HIV infection or immunosuppression, and KS forms very-well demarcated intradermal nodules (Figure 5A). This means that $\mathrm{CO}_{2}$ laser treatment may be easy, fast, and relatively safe. In fact, the procedure was easy and effective, with no serious complications in every patient, with a complete resolution of the lesions, leaving good cosmetic results after two years from treatment (case 1, Figure 4). On the other side, there are some theoretic drawbacks to laser therapy, due to clinical experience, suggesting that the real margins of Kaposi's lesion may be quite distant from the macroscopic one and that the process of wound healing stimulates growth of KS. This could explain late local relapses. It is remarkable that in our patients they followed surgical excision. Vice versa, laser vaporization of excisional borders may enlarge and stabilize margins, as in the laser treatment of HPV infection, to prevent relapses that normally are not far from the primary lesion. In fact, in our opinion, while local relapses may show a disease persistence, relapses far from the surgical area may be due to a new disease.

Owing to the small numbers of patients, no definitive cure is yet known for penile KS in immunocompetent patients. Moreover no follow up schedule is known, even if local relapses may occur within two years from the diagnosis. Our patients did not show any other form of KS (CT scan were negative), but some authors claim that a penile unique lesion could be the first manifestation of a disseminated Kaposi's sarcoma. Therefore, a long follow up is needed, and a gastroscopy may be useful. However, our patients did not undergo gastroscopy owing to the absence of symptoms [15].

In conclusion, primary penile $\mathrm{KS}$ is quite rare in immunocompetent patients. In every case, this disease may be kept in mind when treating nonspecific penile lesions. A histo- 
logic evaluation of the lesion may be useful for planning the right treatment, its clinical staging, and its follow up even if the small number of patients doesn't allow to draw conclusions. In fact, there is no treatment capable of eradicating HHV-8. However, our positive experience shows that it is a slowly progressive disease, as observed by other Authors [2], that may be cured by local therapies, like laser ones.

Key Points

- Kaposi's sarcoma is not always linked to HIV infections

- It may be due to human herpes virus type $8(H H V-8)$ infection

- It may be a slowly progressive disease with a good life expectancy

- It is very rare in immunocompetent patients, however it may be kept in mind when treating nonspecific penile lesion

- Owing to its rarity, no definitive guidelines are found in English literature

- The evidence of effectiveness of any particular intervention is of low quality and does not support recommending any diagnostic and/or therapeutic choice

- $\mathrm{CO}_{2}$ laser therapy might be effective, easy, fast, and safe

\section{Funding}

This article has been published without the support of sponsors.

Conflicts of Interests

The authors declare they have no competing financial interests concerning the topics of this article.

\section{REFERENCES}

1. Mukai MM, Chaves T, Caldas L, et al. Primary Kaposi's sarcoma of the penis. An Bras Dermatol 2009; 84: 524-6; https://doi.org/10.1590/S0365-05962009000500013

2. Rescigno P, Di Trollo R, Buonerba C, et al. Non-AIDS-related Kaposi's sarcoma: a singleinstitution experience. World J Clin Oncol 2013; 4: 52-7; https://doi.org/10.5306/wjco.v4.i2.52

3. Micali G, Nasca MR, De Pasquale R, et al.Primary classic Kaposi's sarcoma of the penis: report of a case and review. J Eur Acad Dermatol Venereol 2003; 17: 320-3; https://doi.org/10.1046/ j.1468-3083.2003.00747.x

4. Chang Y, Cesarman E, Pessin MS, et al. Identification of herpesvirus-like DNA sequences in AIDS-associated Kaposi's sarcoma. Science 1994; 266: 1865-9; https://doi.org/10.1126/ science.7997879

5. Morelli L, Pusiol T, Piscioli F, et al. Herpesvirus 8-Associated Penile Kaposi's sarcoma in an HIV-Negative Patient: First report of a solitary lesion. Am J Dermatopathology 2003; 25: 2831; https://doi.org/10.1097/00000372-200302000-00006

6. Seleit I, Attia A, Maraee A, et al. Isolated Kaposi Sarcoma in two HIV negative patients. $J$ Dermatol Case Rep 2011; 2: 24-6; https://doi.org/10.3315/jdcr.2011.1067

7. Zargari O. Exclusive penile Kaposi's sarcoma: report of an HIV-negative man successfully treated with radiotherapy. J Eur Acad Dermatol Venereol 2006; 20: 318-20; https://doi.org/10.1111/ j.1468-3083.2006.01423.x

8. De Rose AF, Justich M, Mantica G, et al. Kaposi's sarcoma : an unusual penile lesion in a HIV negative patient. Arch It Urol Androl 2017; 89: 164-5; https://doi.org/10.4081/aiua.2017.2.164

9. Kuriyama Y, Shimuzu A, Yamada K, et al. Case of classic Kaposi sarcoma of the penis successfully treated with radiotherapy. J Dermat 2018; 45: e175-e176; https://doi.org/10.1111/13468138.14220

10. Brambilla L, Bellinvia $M$, Tourlaki A, et al. Intralesional vincristine as first-line therapy for nodular lesions in classic Kaposi sarcoma: a prospective study in 151 patients. Br J Dermatol 2010; 162: 854-9; https://doi.org/10.1111/j.1365-2133.2009.09601.x

11. Ghyka G, Alecu M, Halalau F, et al. Intralesional human leukocyte interferon treatment alone or associated with IL-2 in non-AIDS related Kaposi's sarcoma. J Dermatol 1992; 19: 35-9; https://doi.org/10.1111/j.1346-8138.1992.tb03176.x 
12. Celestin Schartz NE, Chevret S, Paz C, et al. Imiquimod 5\% cream for treatment of HIVnegative Kaposi sarcoma skin lesion: a phase I to II, open-label trial in 17 patients. J Am Acad Dermatol 2008; 58: 585-91; https://doi.org/10.1016/j.jaad.2007.11.005

13. Regneir-Rosencher E, Guillot B, Dupin N. Treatments for classic Kaposi sarcoma: a systematic review of the literature. $J$ Am Acad Dermatol 2013; 68: 313-31; https://doi.org/10.1016/j. jaad.2012.04.018

14. Chun YS, Chang SN, Park WH. A case of classical Kaposi's sarcoma of the penis showing a good response to high-energy pulsed carbon dioxide laser therapy.J Dermat 1999; 26: 240-3; https://doi.org/10.1111/j.1346-8138.1999.tb03464.x

15. Kolios G, Kaloterakis A, Filiotou A. Gastroscopic findings in Mediterranean Kaposi's sarcoma (non-AIDS). Gastrointest Endosc 1995; 42: 336-9; https://doi.org/10.1016/S00165107(95)70133-8 